\title{
LA CONDITION DE L'ÉTRANGER DANS LA CONSTITUTION BRÉSILIENNE DE 1988
}

THE STATUS OF ALIENS IN THE 1988 BRAZILIAN CONSTITUTION

Carmen Tiburcio

\begin{abstract}
Professeur de Droit International Privé de l'Université de l'État de Rio de Janeiro - UERJ. Maître et Docteur en Droit International par la Faculté de Droit de l'Université de Virginia, aux États Unis. L'auteur veut remercier la collaboration de Lucas Hermeto pour les recherches et l'aide avec la traduction concernant cet article bien que Jérémie Forquin pour l'aide avec sa révision.

cbtiburc@uninet.com.br
\end{abstract}

Résumé: Cet article examine le traitement de la condition juridique des étrangers à la lumière de la Constitution brésilienne, ainsi que la discipline historique de la matière dans les Chartes constitutionnelles antérieures. Le travail aussi compare la législation en vigueur avec les conventions internationales existantes et avec des principes de droit international, afin de déterminer dans quelle mesure notre Charte actuelle est en conformité avec le droit international.

Mots-clés: Conditión juridique des étrangers au Brésil - Droits de l'homme - Chartes constitutionneles brésiliennes

Abstract: This article examines the treatment of aliens under the Brazilian Constitution, as well as under former Constitutions. This article also compares the Brazilian legislation in force with international treaties and principles of international law, aiming at establishing the extent to which our Constitution is in accordance with international law.

Keywords: Legal status of aliens in Brazil - Human rights - Brazilian Constitutions

\section{INTRODUCTION}

L'objectif de cet article sera d'examiner le traitement des droits des étrangers à la lumière de la Constitution brésilienne, ainsi que la discipline historique de la matière dans les Chartes constitutionnelles 
antérieures. À la fin de ce travail, ces dispositions seront comparées avec les conventions internationales existantes et avec des principes de droit international, afin de déterminer dans quelle mesure notre Charte actuelle est en conformité avec le droit international ${ }^{1}$.

\section{LE CONCEPT D’ « ÉTRANGER »}

Au fil des siècles, la définition d' " étranger » a subi diverses influences : dans l'Antiquité, l'étranger était celui qui n'appartenait pas à la religion de la ville; dans le Moyen Age, le facteur religieux était encore plus remarquable; à partir de l'Age Moderne, spécialement après la Révolution Française, l'étranger passe à être défini en sens négatif : celui qui n'est pas citoyen².

Actuellement, l'étranger est celui qui n'est pas considéré, par les lois d'un État quelconque, comme son national. Chaque pays détermine unilatéralement, conformément à ses lois, ceux qui sont ses nationaux. Étant donné qu'il n'y a pas d'uniformité entre les lois qui traitent de la nationalité dans les différents pays, le phénomène de l'apatridie peut avoir lieu lorsqu'un individu n'est considéré national d'aucun État; il sera par conséquent considéré étranger n'importe où il se trouve. La définition d'étranger, donc, est faite par exclusion.

Dans aucune Constitution, loi, règlement ou n'importe quelle disposition normative brésilienne n'est défini ce qu'est l' " étranger ». La doctrine et la pratique ont créé une définition a contrario sensu à partir du concept de national. Toute personne qui n'est pas nationale brésilienne est considérée un étranger ${ }^{3}$.

Le Brésil ne prévoit pas de différentes catégories d'étrangers ${ }^{4}$; ils sont tous groupés sous la rubrique de « ceux qui n'ont pas la nationalité brésilienne ». La seule exception concerne les nationaux portugais, qui

1 Sur la condition juridique de l'étranger au Brésil sous l'optique du Droit International, v. Carmen Tiburcio, Nationality and the Status of Aliens in the 1988 Brazilian Constitution. In : Jacob Dolinger et Keith S. Rosenn, A Panorama of Brazilian Law 267 (1992).

2 Pour la reconstruction historique plus detaillée de cette trajectoire, v. Carmen Tiburcio, The human rights of aliens under international and comparative law 23-24 (2001).

3 M. Fraga, O novo Estatuto do Estrangeiro Comentado 1 (1985) ; $34^{e}$ Enciclopédia Saraiva do Direito 170 ; A. Dardeau de Carvalho, Situação Jurídica do Estrangeiro no Brasil 9 (1976); J. Afonso da Silva, Curso de Direito Constitucional Positivo 335 (2005), Carmen Tiburcio, A nacionalidade à luz do direito internacional brasileiro. In Carmen Tiburcio e Luís Roberto Barroso, Direito constitutional internacional 245 e ss (2013).

4 Contrairement à ce qui se passe au Royaume-Uni, le Brésil n'a pas de différentes catégories d'individus qui reçoivent la protection de l'État. Depuis l'Acte de Nationalité Britanique de 1981, qui entra en viguer le 1er janvier 1983, l'« étranger » signifie une personne qui n'est pas citoyenne du Commonwealth, British protected person (personne protégée par les britaniques), et de la République de l'Irlande. V. I MacDonald, Immigration Law and Practice in the UK 1 ( $2^{\mathrm{e}}$ éd. 1987). 
jouissent d'une condition spéciale dû à un traité entre le Brésil et le Portugal garantissant la réciprocité de droits aux brésiliens au Portugal ${ }^{5}$. Selon ce traité, les nationaux du Portugal sont assimilés aux brésiliens naturalisés et seulement l'accès à quelques postes officiels qui sont constitutionnellement exclusifs des brésiliens naturels leur est refusé ${ }^{\text {. }}$ Il faut noter que les nationaux d'autres pays peuvent aussi recevoir des droits spéciaux au Brésil par la voie d'un traité, sans que cela n'affecte leur condition d'étranger?'

Il faut observer que le Brésil a, par contre, deux classes de nationaux : ceux qui ont acquis leur nationalité par la naissance (naturels) et ceux qui l'ont acquis par la voie de la naturalisation (naturalisés).

\section{DROITS STATUTAIRES DES ÉTRANGERS}

Les États ont toujours réglementé la situation des étrangers sur leur territoire. En général, leur entrée a toujours été soumise à une série de conditions imposées par les États, en raison de soucis concernant la souveraineté et les intérêts nationaux. Une fois admise la présence de l'étranger sur leur territoire, il fallait toutefois que des droits leurs soient reconnus. Avec le droit humanitaire, il a fallu aussi reconnaître un minimum de droits même aux étrangers qui ne sont pas en situation régulière. Et le commerce international exige aussi que même les étrangers qui ne sont pas sur le territoire national puissent jouir de certains droits, tel que le droit à la propriété privée et à l'accès à la justice.

À la fin du XIXe et au début du XXe siècle, les pays latinoaméricains, tout en essayant d'encourager l'immigration et les investissements, promirent aux étrangers l'égalité de traitement vis-à-vis des nationaux, les mêmes droits civils et économiques leur étant garantis. Cette égalité absolue fut toutefois critiquée par une partie de la doctrine, qui la considérait purement théorique et sans importance pratique ${ }^{8}$, vu que la loi pouvait créer des distinctions entre nationaux et étrangers.

5 Traité de l'Amitié, Coopération et Consulte, entre la République Fédérative du Brésil et la République Portugaise, qui fut incorporé en droit interne par le Decret nº 3.927/2001. Le Brésil et le Portugal ont un lien historique, car le Brésil était une colonie portugaise. V. Moura Ramos, O Novo Direito Português da Nacionalidade. In : Homenagem ao Prof. Antonio Arruda Ferrer Correia 662, no 379 (1986). V. aussi Moura Ramos, La Double Nationalité D'après le Droit Portugais 59. In : Boletim da Faculdade de Direito de Coimbra 203 (1983). Des pays avec une expérience de passée commun aussi peuvent aussi avoir ce type de traitement spécial, tel que le Danemark et l'Islande (Constitution du Royaume de la Danemark de 1953, article 87).

6 Traité d'Amitié, Coopération et Consulte, entre la République Fédérative du Brésil et la République Portugaise, article 14 ; Constitution Fédéral de $1988(\mathrm{CF})$, article $12, \S \S 1^{\text {er }}$ et $3^{\mathrm{e}}$.

7 Par exemple, les nationaux de certains pays paient des impôts plus réduits au Brésil en vertu des traités en vigueur entre le Brésil et ces pays.

8 Edwin Borchard, The Minimus Standard of the Treatment of Aliens. In : American Society of International Law - Proceedings 56, 56 (1939). 
Hormis l'Amérique Latine, cette doctrine n'a pas été reçue avec enthousiasme ${ }^{9}$. L'idée que les étrangers devraient être traités selon un standard minimum international a prévalut. Cette dernière théorie affirme que le droit international a établi un ensemble de droits qui devraient être garantis par tous les pays civilisés sur leur territoire.

Il faut souligner qu'à l'époque où cette doctrine fut créée, il y a eu des difficultés pour l'appliquer, une fois que l'exacte définition du contenu de cet ensemble de droits était imprécise et il n'y avait aucun groupe de droits qui était considéré comme tel. Actuellement, cette difficulté de déterminer des standards internationaux a été significativement réduite, une fois que tout droit de l'homme garantit dans un document international de grande acceptation fait nécessairement partie de cet ensemble ${ }^{10}$, dont le contenu devient donc plus clair avec le développement du droit humanitaire.

Dans le champ du droit positif brésilien, le pouvoir de légiférer en relation à l'immigration et à l'extradition est exclusif du Congrès National ${ }^{11}$. La première législation qui traitait de la situation des étrangers au Brésil fut un décret de 1820 qui interdisait leur entrée au Brésil sans la possession d'un passeport ${ }^{12}$. La Constitution Impérial de 1824 ne contenait que très peu de dispositifs concernant la matière, en établissant seulement que toute personne ne pouvait demeurer ou quitter le pays selon les règlements de police ${ }^{13}$. Il n'y avait aucun dispositif spécifique concernant les étrangers, et le chapitre consacré aux droits individuels ne se référait qu'aux citoyens, différemment de ce qu'on a fait dans les Constitutions postérieures ${ }^{14}$.

9 Alwyn V. Freeman, Recents Aspects of the Calvo Doctrine and the Challenge to International Law, affirme en termes incisifs : "In the guise of liberalism, it reasserts the ancient theory of the unbridled sovereignty, that " states are responsible only to themselfs " leyt us make no mistake about it : there is nothing progressive, humanitarian, or altruistic in the philosophy inspiring this resolution. Its exclusive aim is to free an interested state from restrains imposed by international law upon conduct which would otherwise produce a pecuniary liability to its sister nations. He far-reaching implications of this doctrine are so sinister and so deplorate thta it should be resisted by the profession with every means as it command ".

10 Cet entendement fut premièrement soutenu par F. V. Garcia Amador dans son travail sur la Responsabilité de l'État pour la Comission de Droit International de l'ONU (Sixth Report on International Responsibility - a/cn.4/134 and Add. 1).

$11 \mathrm{CF}$, article 22, XV, qui a réproduit les dispositifs des Constitutions antérieures.

12 Décret du 2 décembre 1820. En fait, puisque le Brésil n'a acquis son indépendence que le 7 septembre de 1822, ledit décret était un acte colonial.

13 Constitution de 1824, article 179, 6 : "l'inviolabilité des droits civils et politiques des citoyens brésiliens, qui a comme base la liberté, la sécurité individuelle et la propriété, est garantie par la Constitution de l'Impère. ".

14 Le titre VIII de la Constitution Impériale s’appellait « des dispositions générales et garanties des droits civils et politiques des citoyens brésiliens » et plusieres alíneas de l'article 179, qui consacrait l'inviolabilité des droits civils et politiques, se référaient à des droits exclusifs des 
En 1889, en vertu de la Proclamation de la République, tous les étrangers présents au Brésil le 15 novembre 1889 furent automatiquement naturalisés ${ }^{15}$. L'obligation d'entrer au pays avec un passeport fut aussi abrogée en $1890^{16}$ et cette règle fut expressément prévue par la Constitution Républicaine de $1891^{17}$. En plus, le texte de 1891 étendit aux étrangers résidents les droits individuels garantis par la Constitution : les droits à la vie, à la sécurité personnelle et à la propriété ${ }^{18}$, ce qui a été suivi par toutes les Chartes constitutionnelles suivantes.

La Constitution de 1934 introduisit le système des quotas pour contrôler l'entrée d'étrangers, en établissant des limites au nombre de nationaux de chaque pays étranger compte tenu de la moyenne de ressortissants de chacun qui avait immigré dans les 50 ans antérieurs ${ }^{19}$. La Charte supprima aussi l'exigence de résidence au pays pour que l'étranger obtienne les garanties constitutionnellement prévues.

Le système de quotas fut maintenu dans la Constitution de 1937. Dû aux tensions mondiales, la sécurité nationale était considérée d'extrême importance et la législation était imprégnée d'un fort sentiment de nationalisme et de crainte de ce qui était étranger. En 1938, le Conseil d'Immigration et Colonisation fut créée pour régler l'immigration d'étrangers vers le Brésil conformément au système de quotas. Il faut noter que ce système ne fut jamais entièrement observé, car beaucoup d'étrangers entraient dans le pays en excédant les quotas permis. Des permissions pour cela étaient données par la législation ordinaire. Enfin, en 1945 fut promulguée une législation contenant des

citoyens, ce qui excluait les étrangers.

15 Décret $\mathrm{n}^{\circ} 58-\mathrm{A} / 1889$.

16 Décret $\mathrm{n}^{\circ} 212 / 1890$.

17 Article 72, § 10 : "La Constitution assure aux brésiliens et étrangers résidents au Pays l'inviolabilité des droits concernant la liberté, la sécurité individuelle et la propriété dans les termes suivants : $\S 10-$ En temps de paix toute personne peut entrer dans le territoire national ou en sortir avec sa fortune et ses biens, quand et de la manière qui lui convient, indépendamment de passeport $»$. Le requisitoire du passeport fut rétablit dans l'Amendement de 1926 à la Constitution de 1891 : «Article 72. La Constitution assure aux brésiliens et étrangers résidents au Pays l'inviolabilité des droits concernant la liberté, la sécurité individuelle et la propriété, dans les termes suivants : $\$ 10$ - En temps de paix toute personne peut entrer au territoire national ou en sortir avec sa fortune et ses biens. ».

18 Constitution de 1891, article 72.

19 Constitution de 1934, article 121, $\S 6^{\circ}:$ : La loi promouvra l'aide de la protection et établira les conditions de travail, dans la ville et dans la campagne, compte tenu de la protection sociale du travailleur et les intérêts économiques du Pays. $\S 6^{\circ}$ - L'entrée d'immigrants dans le territoire national subira les restrictions nécessaires à la garantie de l'intégration ethnique et de la capacité physique et civile de l'immigrant, le courant migratoire de chaque pays ne pouvant pas, toutefois, excéder, annuellement, la limite de deux pour cent sur le nombre total des respectifs nationaux fixés au Brésil au cours des dernières cinquante années ». 
règles concernant les étrangers au Brésil ${ }^{20}$.

Le système de quotas a été éliminé par la Constitution de 1946, dont le texte affirmait que l'entrée et l'installation d'étrangers devraient être établies par la loi. Elle prévoyait aussi la création d'une agence pour superviser des questions d'immigration ${ }^{21}$. Nonobstant ce commandement constitutionnel, cette agence n'a été créée que neuf ans après, lorsque 1'Institut National d'Immigration et Colonisation a été fondé.

Les Constitutions de 1967 et de 1969 ont maintenu le droit des étrangers d'entrer au pays, pourvu que les conditions établies par la législation ordinaire soient remplies ${ }^{22}$. Elles ont aussi réitéré qu'aux étrangers résidents devraient être garantis les mêmes droits fondamentaux que ceux qui l'étaient pour les nationaux ${ }^{23}$. Tous les aspects importants concernant l'entrée et le séjour d'étrangers, ainsi que l'ensemble de leurs droits dans le pays, furent établis par un décret exécutif connu comme « Le Statut de l'Étranger ${ }^{24}$ ». Ce statut demeura en vigueur jusqu'à 1980, quand le Congrès National Brésilien a approuvé une nouvelle Loi d'Immigration ${ }^{25}$. Cette loi, aussi connue comme «Statut de l'Étranger », qui a créé le Conseil National d'Immigration pour coordonner les politiques et actions concernant l'immigration, fut très critiquée par des activistes des droit de l'homme, leaders de l'opposition et par l'Église Catholique, parce qu'elle réduisait significativement le droit de l'étranger de demeurer au pays et les défenses disponibles contre l'expulsion. Ces critiques ont entrainé un amendement constitutionnel promulgué en 1981 qui

20 Decret-loi n 7.967/1945, abrogé par la Loi n 6.815/1980.

21 Constitution de 1946, article 162 : «La sélection, l'entrée, la distribution et la fixation d'immigrants seront soumises, dans la forme de la loi, aux exigences de l'intérêt national. Paragraphe Unique - Un organe fédéral sera chargé d'orienter ces services et de les coordonner avec ceux de la naturalisation et de la colonisation, devant dans les cas de cette dernière considérer les nationaux. "》.

22 Constitution de 1967, article 150, § 26 : «La Constitution assure aux brésiliens et aux étrangers résidents au Pays l'inviolabilité des droits concernant la vie, la liberté la sécurité et la propriété, dans les termes suivants : § 26 - En temps de paix, toute personne pourra entrer avec ses biens dans le territoire national, y démeurer ou en sortir, tout en respectant les prescriptions de la loi ». Constitution de 1969, article 153, § 26 : "La Constitution assurera aux brésiliens et aux étrangers résidents au Pays l'inviolabilité des droits concernant la vie, la liberté, la sécurité et la propriété, dans les termes suivants : $\$ 26-$ En temps de paix, toute personne pourra enter avec ses biens dans le territoire national, y démeurer ou en sortir, tout en respectant les prescriptions de la loi ».

23 Constitution de 1967, article 150; Constitution de 1969, article 153.

24 Decret $n^{\circ} 941 / 1969$. Celle fut la première fois que le Brésil a promulgué une législation qui peut être appelée de « Statut de l'Étranger», car elle incorporait la plupart des législations qui traitait du sujet.

25 Loi n $^{\circ} 6.815 / 1980$. 
a rétabli certaines protections légales aux étrangers dans l'esprit de la tradition légale brésilienne ${ }^{26}$.

\section{ENTRÉE ET SÉJOUR}

D'un côté, le droit du national d'entrer dans le pays de sa nationalité est en même temps un droit individuel et une garantie de l'État d'accueil. De l'autre côté, puisque seulement les nationaux ont le droit inconditionnel d'entrer et de résider dans leur pays, les étrangers peuvent avoir leur entrée refusée. Tous les États souverains exercent un degré de contrôle sur la migration d'étrangers vers leur territoire. La question de quelles limitations sont imposées au pouvoir des États de contrôler l'immigration est assez discutée.

La vision traditionnelle, c'est que le droit international n'impose pas de restrictions sur le pouvoir de l'État de contrôler l'immigration. Cette position est illustrée par la décision de la Cour Suprême des ÉtatsUnis de 1892 qui suit :

It is an accepted maxim in international law, that every sovereign nation has the power, as inherent in sovereignty and esssential to self-preservation, to forbid the entrance of foreigners within its dominions, or to admit them only in such cases and upon such conditions as it may see fit to prescribe. ${ }^{27}$

Le langage utilisé par la Cour Suprême dans ce cas ne laisse pas de doute quant à l'affirmation qu'il n'y a pas, en droit international, de limites pour le pouvoir de l'État en matière de contrôle de l'immigration. En 1889, dans le célèbre cas impliquant des chinois, connu comme "Chinese Exclusion Case », la Cour Suprême Américaine réitéra cette position, en établissant " that the governement of the United States, through the action of the legislative departement, can exclude aliens from its territory is a proposition which we do not think open to controversy $\gg{ }^{28}$ La Cour Suprême alla encore plus loin, en établissant une claire connexion entre le pouvoir d'immigration et la souvérainété nationale, selon les termes suivants :

The power of exclusion of foreigners being an incident of sovereignty belonging to the governement of the United States, as a part of those sovereign powers delegates by Constitution, the right to its

26 Loi n ${ }^{\circ} 6.964 / 1981$.

27 Nishimura Ekiu v. United States, 142 US 651 (1892).

28 Chae Chan Ping v. US, 130 US 581 (1889). 
exercise at any time when, in the judgement of the governement, the interests of the country require it, cannot be granted away or restrained on behalf of any one. ${ }^{29}$

Cette vision traditionnelle est aussi contenue dans l'article $1^{\text {er }}$ de la Convention de la Havane sur la Condition des Étrangers de $1928^{30}$, qui détermine que les États ont le droit d'établir, par la voie de la législation, les conditions générales d'entrée et de séjour d'étrangers dans leur territoire ${ }^{31}$.

Plus récemment, la notion que les États ont le pouvoir illimité d'exclure les étrangers a été questionnée. Il a été soutenu que, sous certaines circonstances, l'État aurait en principe le devoir exceptionnel d'admettre des étrangers, comme dans le cas de résidents permanents, qui ont une expectative légitime d'être réadmis, ou dans les cas de diplomates et de victimes de calamités naturelles (force majeure) ${ }^{32}$. En plus, la norme établissant le non-refoulement ${ }^{33}$ (qui détermine que

29 Chae Chan Ping v. US, 130 US 581 (1889).

30 Incorporée en droit interne, au Brésil, par le Decret n 18.956/1929.

31 Article $1^{\mathrm{er}}$ : "Les États ont le droit d'établir, par moyen des lois, les conditions d'entrée et de résidence des étrangers dans leur territoire ». Les pays l'ayant ratifiée sont : L'Argentine, La Bolivie, Le Brésil, Le Chili, La Colombie, La Costa Rica, Cuba, L'Équateur, Les États-Unis, Le Guatemala, Haiti, Le Honduras, Le Mexique, Le Nicaragua, Le Panama, Le Pérou, La République Dominicaine, Le Salvador, L’Uruguai et Le Venezuela ».

32 R. Plender, International Migration Law 159 (1988).

33 Flávia Piovesan, $O$ direito de asilo e a proteção internacional dos refugiados. In: Nádia de Araújo e Guilherme Assis de Almeida (Coord.), O Direito Internacional dos Refugiados Brasileiros 53-4 (2001) : "le principe du non-refoulement doit être compris aujourd'hui d'une manière plus ample, tout en transcendant les restreints contours conférés par les articles $1^{e r}$ et 33 de la Convention sur le Statut des Réfugiés de 1951. Cet étude défend, ainsi, la nécessité de réduire le domaine du pouvoir discrétionnaire de l'État, afin que des droits universalement assurés soient efectivement respectés. Il convient de dire : au droit de solliciter asile et d'en jouir, énoncé dans la Déclaration Universelle, doit correspondre le devoir de l'État de concéder l'asile. En adoptant la perspective de la protection des droits de l'homme, il est fondamental consolider la théorie de la responsabilité juridique de l'État en ce qui concerne la matière, nonobstant les résistances et les dificultés " ("O principio do non-refoulement deve ser hoje compreendido de forma mais ampla, transcendendo os restritos contornos conferidos pelos artigos $1^{\circ}$ e 33 da Convenção sobre o Estatuto dos Refugiados de 1951. Este estudo defende, assim, a necessidade de reduzir o domínio da discricionariedade do Estado, a fim de que direitos universalmente assegurados sejam efetivamente implementados. Vale dizer, ao direito de solicitar asilo e dele gozar, enunciado na Declaração Universal, há de corresponder o dever do Estado de conceder asilo. Adotando-se a perspectiva da proteção dos direitos humanos, faz-se fundamental consolidar a teoria da responsabilidade jurídica do Estado no tocante à matéria, não obstante todas as resistências e dificuldades "). Dans le même sens, Thamy Pogrebinschi, $O$ direito de asilo e a Constituinte de 1987-88. In: Nádia de Araújo e Guilherme Assis de Almeida (Coord.), O Direito Internacional dos Refugiados Brasileiros 341 (2001). 
l'étranger ne peut pas être remis à un pays où il risque d'être persécuté) pour les réfugiés est souvent citée comme une règle générale de droit international, ayant des effets au-delà de la Convention des Réfugiés ${ }^{34}$. Outre ces règles spécifiques, il y en a d'autres de caractère plus général qui imposent certaines limites à un contrôle étatique sur l'immigration, telles que les règles interdisant la discrimination ${ }^{35}$, le traitement inhumain et dégradant, le traitement arbitraire, ainsi que les règles établissant le droit à la vie familiale ${ }^{36}$.

Enfin, alors que, d'un côté, le droit international garantit aux États la liberté pour établir des règles en ce qui concerne l'immigration, de l'autre, il garantit aux individus certains droits, qui éventuellement peuvent limiter le pouvoir étatique de contrôler l'entrée et l'expulsion d'étrangers.

\section{A. Le contrôle d'immigration au Brésil}

Le contrôle brésilien d'immigration est un procédé composé de deux étapes : premièrement, l'émission d'un visa et, deuxièmement, le contrôle de l'entrée. Ainsi, avant d'émettre le visa, le Consulat brésilien à l'étranger analyse si le requérant remplit tous les conditions du Statut de l'Étranger $^{37}$ et ses règlements, spécialement en relation à son âge ${ }^{38}$, à ses conditions de santé ${ }^{39}$ et aux éventuelles condamnations pénales qui peuvent lui avoir été imposées à l'étranger ${ }^{40}$. Le Consulat vérifie aussi si la présence de l'étranger au Brésil contrarie l'ordre public ou les intérêts brésiliens ${ }^{41}$. Au moment de l'entrée, les étrangers sont

34 G. Goodwin-Gill, International Law and the Movement of Persons Between States 137 (1978); G. Goodwin-Gill, The Refugee in International Law 69 (1983). À la préface du premier livre, l'auteur a écrit : "The central thesis of this work is the competence clearly limited and confined by established and emergent rules and standards of international law $»$.

35 Il convient de rappeler que l'État ne peut pas discriminer une nationalité, race ou réligion spécifique, ou nier l'entrée, e. g., aux femmes, sans aucune justification raisonable, dès que cela heurterait la règle de droit international interdisant la discrimination. Carmen Tiburcio, The Human Rights of Aliens under International and Comparative Law 216 (2001).

36 Oppenheim enfatisa quelques-uns de ces points : "Although a State may exercise its right of expulsion according to discretion, it must not abuse its right by proceeding in an arbitrary manner " ("Bien qu'un État puisse exercer son droit d'exlpulsion de manière discrétionnaire, il ne faut pas abuser de son droit en procédant de manière arbitraire ».). L. Oppenheim, International Law 691 (1955).

37 Loi n $^{\circ} 6.815 / 1980$, modifié par la Loi no 6964/1981 (Statut de l'Étranger).

38 Aucun étranger mineur de dix-huit ans n'aura droit au visa pour entrer dans le pays s'il voyage seule, sans la compagnie d'un responsable ou sans autorisation due. Statut de l'Étranger, article 7, I.

39 Statut de l'Étranger, article 7, V.

40 Statut de l'Étranger, article 7, IV.

41 Statut de l'Étranger, article 7, II. 
réexaminés pour qu'il soit garanti qu'il n'y ait pas eu de changement de circonstances qui fassent que son admission devienne indésirable ${ }^{42}$. L'émission du visa ne donne pas à l'étranger la garantie d'entrée au pays, car celle-ci peut encore lui être refusée ${ }^{43}$.

\section{B. Types de Visa}

La condition générale d'entrée au Brésil, c'est que l'étranger possède un passeport et un visa d'entrée ${ }^{44}$. Les Consulats Brésiliens émettent sept types de visa ${ }^{45}$ : (1) le visa de transit, qui permet à l'étranger un passage par le territoire national ${ }^{46}$; (2) le visa de touriste, valable pour un séjour de trois mois ${ }^{47}$; (3) le visa temporaire, pour un séjour limitét8 ; (4) le visa permanent, pour un étranger qui veut y demeurer $\mathrm{d}^{\prime}$ une façon permanente ${ }^{49}$; (5) le visa de courtoisie ; (6) le visa officiel ; et (7) le visa diplomatique ${ }^{50}$. Ces deux derniers sont des types de visas émis par le Ministre des Relations Extérieurs aux représentants de gouvernements étrangers, aux membres d'organisations internationales, aux diplomates étrangers et à leurs familles. Théoriquement, le visa ne peut être sollicité qu'à l'étranger. Des exceptions à cette règle, toutefois, sont admises en ce qui concerne la conversion de visas ${ }^{51}$. La législation brésilienne exige aussi que les étrangers s'enregistrent au pays pour recevoir leur carte d'identité 52 .

42 Statut de l'Étranger, article 22.

43 La Constitution de 1988 répète un dispositif consacré par la tradition du droit brésilien garantissant aux étrangers la liberté d'entrer et de quitter le pays, pourvu que les conditions établies dans la législation ordinaire soient remplies (article 5, XV). V. aussi Statut de 1 Étranger, article 26 .

44 Il y a une exception législative quant aux nationaux de pays voisins, qui ont la permission pour entrer au Brésil en portant seulement leur carte d'identité. Statut de l Étranger, article 21. 45 Statut de l'Étranger, article 4.

46 Le Brésil n'exige pas de visa à ceux qui passent par le territoire national (transit) en route vers un autre pays, sauf si une escale est faite au Brésil. Statut de l'Étranger, article 8, § 2.

47 Les étrangers n'ont pas besoin de visa de touriste s'ils sont nationaux d'un pays ayant un accord de réciprocité avec le Brésil. Statut de l'Étranger, article 10.

48 Ce visa, émis pour une durée variable, est utilisé par des « hommes d'affaires », journalistes, étudiants, artistes et tous qui ont une mission au Brésil pendant une durée de temps limitée. Statut de l'Étranger, articles 13 et 14.

49 Ce visa est en général concédé aux étrangers qui veulent s'instaler au pays pour des raisons familiales ou pour travailler dans des régions où leurs services sont particulièrement nécessaires. 50 Statut de 1'Étranger, article 19: « Le Ministère des Rélations Extérieures définira les cas de concession, prorrogation ou dispense des visas diplomatiques, officiel ou de courtoisie ».

51 Les étrangers qui reçoivent certains types de visa temporaires, diplomatiques ou officiels ont la permission pour les transformer au Brésil en visa temporaire ou permanent. Statut de l'Étranger, articles 37 et 39.

52 Le registre n'est obligatoire que pour ceux qui sont entrés dans le pays comme résidents 


\section{Abrogation de LA PERMisSion de SÉJOUR ET DE RÉSIDENCE}

Les États ont le droit d'expulser de leur territoire les étrangers indésirables. Comme limite à cette prérogative, le droit international détermine que l'expulsion ${ }^{53}$ doit être permise par la législation et doit être fondée sur une décision en conformité avec la loi ${ }^{54}$. Des obligations sont aussi imposées aux États quant à la manière d'effectuer l'expulsion, la cruauté étant interdite et l'avis avec une antécédence raisonnable étant obligatoire, par exemple ${ }^{55}$.

L'extradition ${ }^{56}$ et la déportation sont d'autres mesures que l'État peut prendre pour contraindre des étrangers à quitter leur pays de résidence. Les deux divergent de l'expulsion parce que dans celle-ci il y a l'utilisation d'un pouvoir discrétionnaire, alors que dans les deux autres le critère est plus objectif ${ }^{57}$. La déportation ${ }^{58}$ en général se tient

permanents, résidents temporaires ou réfugiés. Statut de 1 Étranger, article. 30.

53 Selon la définition de Celso de Albuquerque Mello (Direito Internacional Público 619, vol 2 1979), "l'expulsion est l'acte politique-administratif qui oblige l'étranger à quitter le territoire national, dans lequel il ne peut pas retourner " (" expulsão é o ato político-administrativo que obriga o estrangeiro a sair do território nacional, ao qual não pode mais voltar »). José Afonso da Silva (Curso de Direito Constitucional Positivo 342, 2005) la définit comme « un moyen coactif pour rétirer l'étranger du territoire national pour un délit, une infraction ou des actes qui le font devenir incovénient" (" um modo coativo de retirar o estrangeiro do território nacional por delito ou infração ou atos que o tornem inconveniente »). Jacob Dolinger (Direito Internacional Privado : Parte Geral 126, 2014) positionne cet institut comme «le procédé par laquelle un pays expulse de son territoire un étranger résident, en raison d'un crime pratiquée ou d'un comportement nocif pour les intérêts nationaux, le retour au pays duquel il a été expulsé lui étant interdit (" o processo pelo qual um país expele de seu território estrangeiro residente, em razão de crime ali praticado ou de comportamento nocivo aos interesses nacionais, ficando-lhe vedado o retorno ao país donde foi expulso ».).

54 Goodwin-Gill, International Law and the Movement of Persons Between States 263 (1978), qui cite Whiteman e Hackworth.

55 Dans l'Affaire Dillon, de 1928, devant la Mexico-US General Comission, le Commissionnaire Nielsen a observé que le droit souverain à l'expulsion n'était pas nié aux États-Unis, mais la plainte était fondée sur la manière de l'expulsion. Dans l'Affaire Gourrier (1868), un national américain expulsé du Mexique a reçu une indemnisation, bien que le procédé d'expulsion ait été fait conformement à la loi, en raison de l'inutile cruauté de l'acte. V. Goodwin-Gill, International Law and the Movement of Persons Between States 276-77 (1978).

56 Sur ce sujet, v. Carmen Tiburcio, Extradição no Brasil : análise à luz da jurisprudência e do direito comparado. In: Carmen Tiburcio e Luís Roberto Barroso, Direito constitucional internacional 321 e ss (2013).

57 V. Carlos Mário da Silva Velloso, O Direito Internacional e o Supremo Tribunal Federal 6-7. In: Revista de Direito Administrativo, v. 229, jul.-set./2002.

58 José Afonso da Silva (Curso de Direito Constitucional Positivo 342, 2005) définit la déportation comme "la sortie compulsoire de l'étranger. Elle se fonde sur le fait que l'étranger entre ou demeure irrégulièrement dans le territoire national (article 5, XV)» (« saída compulsória do estrangeiro. Fundamenta-se no fato de o estrangeiro entrar ou 
lorsqu'un individu entre illégalement au pays ou dépasse la période permis par le visa ; l'extradition ${ }^{59}$ concerne le procédé de remise d'un individu, accusé ou condamné pénalement dans un État autre que celui de sa résidence, à une autre juridiction, compétente pour connaître de l'infraction qui est réputée commise par lui. En plus, l'étranger peut retourner au pays après avoir été déporté, à condition qu'il ait régularisé sa situation, alors que l'extradition présuppose la pratique d'une infraction pénale, ce qui met l'individu dans la condition de malfaiteur. Il faut souligner qu'il y a des États qui admettent l'extradition de leurs nationaux. En raison de cela, des instruments de protection aux droits de l'homme ne font pas référence à des garanties concernant l'extradition et la déportation, mais le font quant à l'expulsion ${ }^{60}$. Toutefois, cela ne

permanecer irregularmente no território nacional.»). José Francisco Rezek, Ministre en retrait du STF (Direito Internacional Público: Curso elementar 187, 2002) définit l'institut comme « une forme d'exclusion, du territoire national, de l'étranger qui s'y trouve après une entrée irrégulière - en général clandestine -, ou dont le séjour est devenu irregulier - presque toujours par l'excès de délai, ou par exercice de travail rémunéré dans le cas du touriste ( uma forma de exclusão, do território nacional, daquele estrangeiro que aqui se encontre após uma entrada irregular - geralmente clandestina -, ou cuja estada tenha-se tornado irregular - quase sempre por excesso de prazo, ou por exercicio de trabalho remunerado, no caso do turista ». Jacob Dolinger (Direito Internacional Privado : Parte Geral 126-127, 2014, de son côté, définit la déportation comme «le procédé de dévolution de l'étranger qui y arrive ou demeure irrégulièrement vers le pays de sa nationalité ou de sa provenance. Alors que dans l'expulsion le retrait se fait à cause d'une pratique ayant eu lieu après l'arrivée et la fixation de l'étranger au territoire du pays, la déportation trouve son origine exclusivement de son entrée ou séjour irrégulièrs dans le pays » (" o processo de devolução de estrangeiro que aqui chega ou permanece irregularmente para o país de sua nacionalidade ou de sua procedência. Enquanto na expulsão, a remoção se dá por prática ocorrida após a chegada e a fixação do estrangeiro no território do país, a deportação se origina exclusivamente de sua entrada ou estada irregular no país. ").

59 José Francisco Rezek (Direito Internacional Público: Curso elementar 189, 2002) définit l'extradiction comme « la remise, par un État à un autre, à la demande de celui-ci, de l'individu qui, en son territoire doit répondre à un procès pénal ou à y accomplir une peine » ("a entrega, por um Estado a outro, e a pedido deste, de indivíduo que em seu território deva responder a processo penal ou cumprir pena »). Celso de A. Mello (Direito Internacional Público 601, v. 2, 1979) affirme qu'il s'agit de « l'acte par le biais duquel un individu est remis par un État à un autre, qui soit compétent afin d' instruir un procès contre lui ou de le punir » (" o ato por meio do qual um individuo é entregue por um Estado a outro, que seja competente a fim de processálo e puni-lo »). De son côté, Jacob Dolinger (Direito Internacional Privado : Parte Geral 126, 2014) 1'a définit comme «le procédé par lequel un État accorde la demande d'un autre État, en lui remettant la personne poursuivie au pays sollicitant pour un crime puni dans la législation des deux pays, les nationaux du pays sollicité ne pouvant pas, en règle générale, être extradés ». (" o processo pelo qual um Estado atende ao pedido de outro Estado, remetendo-lhe pessoa processada no país solicitante por crime punido na legislação de ambos os países, não se extraditando, via de regra, nacional do país solicitado. »).

60 Nonobstant le fait que certaines garanties, telles que le droit à la vie, à l'intégrité physique 
se passe pas avec le Brésil, dont la Constitution interdit expressément l'extradition de nationaux, ainsi que d'étrangers accusés d'avoir commis un crime politique ou d'opinion ${ }^{61}$.

En règle générale, une décision administrative ou judiciaire n'est pas suffisante pour que la permission pour l'étranger de demeurer au pays soit abrogée, la sortie forcée des permanents exige un procès administratif ou judiciaire préalable. La permission pour demeurer au pays finit automatiquement lorsque le visa s'expire sans rénovation. Après cette date, la permanence de l'étranger au pays devient illégale.

Le séjour d'un étranger au Brésil ne peut être interrompu involontairement que par les procédures de déportation, d'expulsion et d'extradition ${ }^{62}$. La déportation et l'expulsion sont des procédés administratifs, alors que l'extradition est un procédé judiciaire. L'acte de déporter implique l'éloignement d'un individu du pays, soit en raison de son entrée illégale, soit en raison de l'expiration du visa, soit par la désobéissance d'un dispositif du Statut de l'Étranger (par exemple, travailler sans permission ${ }^{63}$ ). L'expulsion se fonde sur des raisons différentes de celles qui sont nécessaires pour donner lieu à la déportation et exige une enquête préalable du Ministre de la Justice ${ }^{64}$ dans laquelle sont garantis à l'étranger le droit de la défense ${ }^{65}$. Comparées avec celles aux États-Unis, les bases pour l'expulsion au Brésil sont assez générales : comportement qui contrarie l'ordre politique ou social, la moralité et la tranquillité, ou l'économie populaire ${ }^{66}$.

Puisque la loi brésilienne a toujours essayé de préserver l'unité familiale, un étranger qui a une famille stable au Brésil en général ne peut en être expulsé, car la règle au Brésil c'est que la famille a le droit de demeurer unie ${ }^{67}$. Le Statut de l'Étranger, actuellement, interdit

et à la vie familiale, sont aussi tûtelées dans le contexte de l'extradiction.

61 Constitution de 1988, article 5, LI et LII : « Tous sont égaux devant la loi, sans distinction de n'importe quelle nature que ce soit, aux brésiliens et aux étrangers résidant au pays étant garantie l'inviolabilité du droit à la vie, à la liberté, à l'égalité, à la sécurité et à la propriété, dans les termes suivants : LI - aucun brésilien ne sera extradé, sauf le naturalisé, en cas de crime commum, pratiqué avant la naturalisation, ou de participation prouvé à un trafic illicite de stupéfiants et drogues semblables, dans la forme de la loi ; LII - l'extradition de l'étranger ne sera pas accordée en cas de crime politique ou d'opinion ».

$62 \mathrm{~V}$. Gilmar Mendes, Direito de Nacionalidade e Regime Jurídico do Estrangeiro 13. In: Direito Público, $\mathrm{n}^{\circ} 14$, out.-dez./2006.

63 Statut de l'Étranger, article $57 \S 1^{\circ}$.

64 Statut de l'Étranger, article 70.

65 STF, DJU 29 nov. 1996, HC 73.940/SP, Rel. Min. Maurício Corrêa.

66 Statut de l'Étranger, article 65. Il y a aussi d'autres bases pour l'expulsion, telles que la fraude lors de l'entrée au pays ou le refus de le quitter après l'expiration du visa. Dans les cas où la déportation n'est pas possible, l'expulsion peut avoir lieu en raison de vagabondage ou de l'inobservance d'un dispositif du Statut de l'Étranger.

67 Jacob Dolinger, Das Limitações ao Poder de Expulsar Estrangeiros. In: Estudos em 
l'expulsion d'un étranger marié avec un national brésilien il y a plus de cinq ans ${ }^{68}$ ou qui a la garde d'enfants brésiliens qui dépendent financièrement de lui ${ }^{69}$. Le STJ a récemment flexibilisé cette règle et $\mathrm{n}^{\prime}$ exige plus la dépendance financière ${ }^{70}$.

Tout étranger a le droit de questionner devant les tribunaux brésiliens la validité de toute action qui peut résulter de sa sortie forcée du pays, que ce soit par l'expulsion ou par la déportation ${ }^{71}$. Le remède juridique disponible est l'habeas corpus et il s'adresse au pouvoir judiciaire, vu que le Brésil adopte le système d'unicité de juridiction. Toutefois, il est très commun que l'individu revendique son droit de demeurer au Brésil par le biais d'un mandat de sécurité (" mandado de segurança »). Dans ce cas, le Suprême Tribunal Fédéral (STF), qui a la compétence originale pour connaître de ces demandes ${ }^{72}$, décide souvent par la conversion du mandat de sécurité en habeas corpus ${ }^{73}$.

La demande d'extradition est jugée uniquement par le $\mathrm{STF}^{74}$ et elle doit être fondée sur un traité d'extradition entre le Brésil et l'État requérant ou sur une promesse de réciprocité ${ }^{75}$. La décision du STF en relation à la demande d'extradition est définitive et ne peut pas faire l'objet d'un recours. En plus, la question de liens familiaux dans le pays n'a pas d'interférence en matière d'extradition ${ }^{76}$.

Les étrangers peuvent quitter le Brésil volontairement à

Homenagem ao Professor Haroldo Valladão (1983).

$68 \mathrm{La}$ condition de la période minimum de 5 ans de mariage a été introduite pour éviter des mariages frauduleux.

69 Statut de 1'Étranger, aticle 75. V. aussi Compilation de jurisprudence ${ }^{\circ} 1$ du STF (approuvée em Session Pleine le 13/12/1963) : "Est interdite l'expulsion d'un étranger marié avec une brésilienne, ou qui a un enfant brésilien, dépendant de l'économie paternelle. ».

70 STJ, DJU 23.avril.2010, HC 141642/DF, Rel Min. Benedito Gonçalves.

71 Constitution de 1988, article 5, XXXV.

72 Constitution de 1988, article 102, I, d.

73 STF, DJU $1^{\circ}$ mar. 1996, HC 72.082/RJ, Rel. Min. Francisco Rezek: « ... le moyen procédural adéquat pour contester le décret d'expulsion est l'Habeas Copus. Ainsi s'est fondée la juisprudence du Suprême [Tribunal Fédéral], soit parce que la personne expulsée est en détention, soit parce qu'il s'agit du remède le plus rapide. " ("...o meio processual adequado para se impugnar decreto expulsório é o Habeas Corpus. Assim se firmou a jurisprudência do Supremo, seja porque o expulsando via de regra está preso, seja porque se trata de remédio mais expedito. ").

74 Constitution de 1988, article 102, I, g. (STF, DJU 22.mar.2011, HC 101.528/PA, Rel. Min. Dias Toffoli). Aussi STF, DJU 20.ago, 2010, HC 101.269/DF, Rel. Min. Cármen Lúcia.

75 Statut de l'Étranger, article 76.

76 STF, DJU 6 jul. 1964, Compilation de jurisprudence $n^{\circ}$ 421, Tribunal Plein. Dans le même sens, STF, DJU 13 avr. 2007, Ext 997/Alemanha, Rel. Min. Joaquim Barbosa; STF, DJU 7 déc. 2006, Ext 967/Bélgica, Rel. Min. Ricardo Lewandowski; STF, DJU 17 nov. 2006, Ext 984/ Estados Unidos, Rel. Min. Carlos Britto; STF, DJU 10 août. 2006, Ext 995/Espanha, Rel. Min. Carlos Britto. 
n'importe quel moment (pourvu qu'ils ne soient accusés d'avoir commis un crime au Brésil et qu'ils n'accomplissent pas une peine de prison) ; ce droit est garanti par la Constitution Brésilienne ${ }^{77}$ et par le Statut de l'Étranger ${ }^{78}$. Il n'est pas nécessaire qu'ils obtiennent un visa pour cela. Lorsqu'il quitte le pays, la Constitution garantit que l'étranger est libre $\mathrm{d}^{\prime}$ emporter avec lui tous ses biens ${ }^{79}$.

\section{GARANTIES JURIDIQUES GÉNÉRALES}

\section{A. Droits de l'homme et droits fondamentaux des étrangers}

Les étrangers résidants au Brésil jouissent des mêmes droits à la vie, à la sécurité et à la propriété que ceux qui sont garantis aux nationaux selon les termes de la Constitution en vigueur et conformément à toutes les Chartes constitutionnelles depuis la première Constitution Républicaine, de 1891. En plus, tous les droits fondamentaux conférés par la Constitution de 1988 dans les alinéas de l'article 5 sont aussi garantis aux étrangers résidents, tels que le droit à l'égalité de traitement, le droit de ne pas être torturé, la liberté d'expression, le droit à la vie privée, le droit au libre exercice de toute profession, le droit d'association, le droit à la propriété privée, le droit d'accès à la justice, ainsi que les droits à l'assistance sociale ${ }^{80}$ et à l'éducation ${ }^{81}$, parmi d'autres. Le fait que la Constitution ne garantisse tous ces droits qu'aux étrangers résidents, toutefois, ne signifie pas que les non-résidents ne soient pas protégés par le système légal brésilien ${ }^{82}$. En divers moments, le STF a étendu plusieurs droits établis dans la Constitution à tous les étrangers, y compris les touristes et même, dans certains cas, à ceux qui ne sont pas dans le pays, tels que l'accès à la justice, le droit à la propriété privée et la droit à la protection à la propriété intellectuelle.

La garantie de droits fondamentaux à tout étranger non-résident n'a pas toujours été reconnue au Brésil. L'un des premiers cas examinés

77 Constitution de 1988, article 5, XV.

78 Statut de l'Étranger, aticle 50.

79 Constitution de 1988, article 5, XV.

80 Constitution de 1988, article 203.

81 Constitution de 1988, article 205.

82 Celso Bastos, Curso de Direito Constitucional 178 (1999). L'auteur souligne que la Constitution étend les droits fondamentaux à toute pesonne qui se trouve sur le territoire brésilien ; il conclue que les auteurs de la Constitution, lorsqu'ils ont fait référence aux étrangers résidents, n'ont pas utilisé l'expression dans son sens authentique, c'est-à-dire le fait d'être légalement domicilié au Brésil, mais ils ont eu l' intention d'étendre ces doits à toute personne qui se trouve physiquement au Brésil, ce qui signifie toute personne qui est en contact avec notre ordre juridique. 
par nos Cours, après la Constituions de 1891, a été un habeas corpus au nom de la famille royale portugaise, qui avait été bannie du Brésil à la suite de la Proclamation de la République. Le cas ayant été décidé par nos tribunaux fédéraux en 1903, on soutient que la Constitution de 1891 garantissait les droits établis dans l'article $72^{83}$ seulement aux étrangers résidents. Puisque la famille impériale ne résidait plus au Brésil et puisque l'habeas corpus était l'un des droits établis dans ce dispositif, les tribunaux ont conclu que la famille impériale n'avait pas droit à l'habeas corpus ${ }^{84}$.

Une partie de la doctrine soutient que l'article de la Constitution qui garantit les droits fondamentaux est une norme générique et que les dispositifs suivant cet article pourraient étendre ces droits aux étrangers non-résidents ou bien les nier aux étrangers résidents. Quelques-uns de ces droits, par contre, devraient être garantis à tout être humain ${ }^{85}$.

D'autres auteurs font une interprétation littérale de la Constitution lorsqu'elle affirme que les droits ne sont garantis qu'aux étrangers résidents, mais ils concluent que les non-résidents ont aussi cette garantie en raison des conventions internationales de droit de l'homme dûment ratifiées par le Brésil et qui garantissent ces droits à toutes les personnes ${ }^{86}$.

83 « La Constitution assure aux brésiliens et aux étrangers résidents au Pays l'inviolabilité des droits concernant la liberté, la sécurité individuelle et la propriété (...) ».

84 Habeas Corpus n ${ }^{\circ}$ 1.973, 91 O Direito 414, 434 (1903).

85 Pontes de Miranda, 4 Comentários à Constituição de 1967 com a Emenda $n^{\circ} 1$ de 1969 695-6 (1974) et Manoel Gonçalves Ferreira, Direito humanos fundamentais 29 (2004). Dans le même sens, Jacob Dolinger, Comentários à Constituição de 1988114 (1990), classifie les droits fondamentaux en génériques, qui sont garantis à tous, spécifiques, garantis à tout brésilien et étranger résident, et restreints, qui ne sont garantis qu'aux brésiliens. V. aussi Guido Fernando Silva Soares, Os Direito Humanos e a proteção dos estrangeiros 185. In: Revista de Informação Legislativa, n 164, abr.-jun./2004. Dans le même sens, STF, DJU 6 avr. 2001, Ext 633/China, Rel. Min. Celso de Mello.

86 José Afonso da Silva, Curso de Direito Constitucional Positivo 335 (2005); Baptista, O Estrangeiro: Reflexões para a Constituinte. In: A nova Constituição e o Direito Internacional 135, 137 (J. Dolinger ed. 1987). Dans le même sens, José Afonso da Silva, Comentário contextual à Constituição 65 (2006) : «Si la Constitution indique les destinataires de ces droits, cela doit avoir des conséquences normatives. Cela ne veut pas dire que les étrangers nonrésidents, lorsqu'ils se trouvent régulièrement sur le territoire national, peuvent être soumis à l'arbitre et ne disposent d'aucun moyen - y compris les jurisditionnels - pour régler les situations subjectives. Pour les protéger, il y a d'autres normes juridiques, y compris de Droit International, lesquelles doivent être respectées et observées par le Brésil et ses autorités, de même qu'il existe des normes légales, traduites dans de législation spéciale, qui définissent les droits et la condition juridique de l'étranger non-résident qui serait régulièrement entré au territoire brésilien » ("Se a Constituição aponta os destinatários desses direitos, isso há de ter conseqüências normativas. Isso não quer dizer que os estrangeiros não-residentes, quando regularmente se encontrem no território nacional, possam sofrer o arbitrio e não disponham de qualquer meio - incluindo os jurisdicionais - para tutelar situações subjetivas . Para 
Il y a encore un troisième courant qui considère que l'expression « résidents au Brésil » doit être interprétée au sens que la Constitution ne peut assurer les droits fondamentaux que sur le territoire national. Ainsi, la norme n'exclut pas de son application les étrangers en transit, par exemple ${ }^{87}$. Or, les droits fondamentaux ont un caractère universel, en s'adressant à tous qui se trouvent sous la tutelle de l'ordre juridique brésilien, dans les limites de la souveraineté nationale, indépendamment de leur nationalité ${ }^{88}$. Beaucoup d'auteurs considèrent en plus que celleci est la meilleure interprétation, compte tenu des règles des articles $3^{\circ}$, alinéa IV et $9^{\circ}$, alinéa II de la Constitution Fédérale.

On débat aussi en doctrine la possibilité ou non pour la législation ordinaire de créer des nouvelles distinctions entre les étrangers résidents et les nationaux. Quelques auteurs croient que, hors les distinctions faites dans le texte constitutionnel et les concessions offertes par la Constitution à la législation ordinaire, aucune autre distinction n'est possible. D'autres auteurs adoptent une position différente ${ }^{89}$. Ceux qui affirment que la législation ordinaire ne peut pas discriminé les étrangers résidents sans autorisation expresse de la Constitution fondent leur position sur le principe qui explicitement interdit la discrimination fondée sur l'origine de l'individu, ce qui est l'un des objectifs de la

protegê-los há outras normas jurídicas, inclusive de Direito Internacional, que o Brasil e suas autoridades têm que respeitar e observar, assim como existem normas legais, traduzidas em legislação especial, que definem os direitos e a condição jurídica do estrangeiro não-residente que tenha ingressado regularmente no território brasileiro »).

87 Alexandre de Moraes, Direito Constitucional 29-30 (2006); Kildare Gonçalves Carvalho, Direito Constitucional 587 (2007). Dans le même sens, STF, RTJ 3/566-68, RE n 33.919/ DF, Rel. Min. Cândido Mota Filho : l'expression 'résidents au pays' se réfère tantôt aux étrangers, tantôt aux brésiliens, et signifie seulement que les droits et garanties individuelles (...) sont assurés (..) dans les limites de la souverainété territoriale nationale. Il est évident que la Constitution ne pourrait pas assurer à des étrangers et brésiliens hors du pays (...) la validité et la jouissance des droits et garanties. (...) Pourvu, toutefois, qu'il s'agisse d'un acte d'autorité brésilienne et que le remède constitutionnel s'adresse à produire des résultats dans le Pays, peu importe que l'impétrant y réside ou non" ("A expressão 'residentes no País tanto se refere a estrangeiros como a brasileiros e significa tão somente que os direitos e garantias individuais (...) são assegurados (...) dentro dos limites da soberania territorial nacional. Óbvio que a Constituição não poderia assegurar a estrangeiros ou brasileiros fora do país (...) a validade e gozo dos direitos e garantias. (...) Desde porém, que se trate de ato de autoridade brasileira e se destine o remédio processual a produzir resultados dentro do País, pouco importa que o impetrante resida aqui ou não »). Selon le vote du Ministre Rapporteur, « si nous reconnaissions qu'au Pays sont abolis les droits des étrangers non-résidents, nous commettrions une violence » ( "se reconhecêssemos que no País ficam extintos os direitos dos estrangeiros não residentes, cometeríamos uma violência »).

88 Kildare Gonçalves Carvalho, Direito Constitucional 588 (2007).

89 V. O. Tenório, Direito Internacional Privado, v. 1, 268, 269 (1976); W. Campos Batalha, Direito Internacional Privado, v. 2, 28, 29 (1977). 
République Fédérative du Brésil d'après le texte constitutionne ${ }^{90}$. De la même manière, l'article 5 de la Constitution garantit, en termes généraux, aux brésiliens et aux étrangers résidents, le droit à la vie, à la liberté, à l'égalité, à la sécurité et à la propriété; ces droits sont la base pour tous les droits fondamentaux ${ }^{91}$. Puisque les étrangers ont le droit d'être également traités ${ }^{92}$, la législation ordinaire qui établit un traitement discriminatoire sans autorisation expresse de la Constitution devrait être considérée inconstitutionnelle ${ }^{93}$.

La distinction entre nationaux et étrangers, pour d'autres auteurs, outre les limites fixées par la Constitution, trouve aussi des restrictions dans les traités et conventions internationales sur les droits et garanties de la personne humaine dont le Brésil fait partie. On peut dire qu'il s'agit de normes du Droit International de la Personne Humaine, dans lesquelles on trouve le droit d'asile et le droit des réfugiés ${ }^{94}$.

Le même argument peut être utilisé en relation avec le droit du travail, garanti aux brésiliens et aux étrangers résidents ${ }^{95}$. Quelques auteurs soutiennent que toute législation établissant des limitations au droit de l'étranger au travail est inconstitutionnelle ${ }^{96}$. Par conséquent, les normes qui conditionnent l'exercice d'une profession à la réciprocité seraient inconstitutionnelles ${ }^{97}$, ainsi que tous les dispositifs du Statut de l'Étranger interdisant l'exercice de fonctions non expressément mentionnées dans la Constitution ${ }^{98}$, toutes les interdictions d'exercer une profession, telles que celle d'interprète public ${ }^{99}$, d'expéditeur en douane ${ }^{100}$ et d'agent d'assurances ${ }^{101}$ et le dispositif de la Consolidation des Lois du Travail (CLT) ${ }^{102}$ qui exige que deux tiers de l'ensemble des travailleurs soient nationaux brésiliens ${ }^{103}$.

90 Constitution de 1988, article 3, IV.

91 M. Gonçalves Ferreira Filho, Comentários à Constituição Brasileira 586 (5 ed. 1984).

92 Constitution de 1988, article 5, I.

93 Dans ce sens, v. STF, DJU 19 dez. 1997, RE 161.243/DF, Rel. Min. Carlos Velloso.

94 Guido Fernando Silva Soares, Os Direitos Humanos e a proteção dos estrangeiros 198. In:

Revista de Informação Legislativa, $\mathrm{n}^{\circ}$ 162, abr.-jun./2004.

95 Constitution de 1988, article 5, XIII.

96 Cette position est soutenue par l'auteur de cet article. Dans le même sens, H. Valladão, Direito Internacional Privado, v. 1, 424 (1980); Soares, Os estrangeiros e as Atividades a eles Vedadas ou Restringidas Proibição Constitucional a Discriminação pela Lei Ordinária de Brasileiros Naturalizados. In : A Nova Constituição e o Direito Internacional 118, 123 (1987). 97 Statut de l'Ordre des Avocats du Brésil, Lei no 4.215/1963, aticles 48, 49 et 51. Cette loi fut abrogée par la Loi no 8906/1994, qui ne fait pas référence à la question de la réciprocité.

98 Statut de l'Étranger, article 106, VI, VII, VIII e IX.

99 Decret $n^{\circ} 13.609 / 1943$, article 3, c.

100 Decret $\mathrm{n}^{\circ} 4.014 / 1942$, article 19 .

101 Loi $^{\circ} 4.594 / 1964$, article 3 , a, $\S 1^{\circ}$.

102 Consolidation des Lois du Travail, article 354.

103 Alors que la Constitution de 1969, article 165, XII admitait expressément la proportionnalité 
Cette discussion se déroule depuis le début du XXe siècle, lorsque la question sur la constitutionalité de l'expulsion d'étrangers résidents fut suscitée, en raison de l'article 72 de la Constitution de 1891 et de son silence en relation à l'expulsion. Pedro Lessa, Ruy Barbosa et Germano Hasslosher croyaient que, puisque la Constitution établissait des droits tout à fait égaux pour les étrangers résidents et pour les nationaux, et comme ceux-ci ne pouvaient pas être expulsés, les étrangers résidents aussi ne le pourraient pas, à moins qu'il y eût une autorisation expresse dans la Constitution. ${ }^{104}$

\section{B. Capacité des étrangers}

En principe, chaque État détermine l'étendue de la capacité juridique des personnes qui se trouvent sur son territoire. D'après la doctrine :

"Le pouvoir de déterminer les droits et les devoirs dont chaque individu peut avoir la jouissance; en d'autres termes, l'étendue de la personnalité juridique de chacun, relève de la souveraineté des États. Ce pouvoir n'est que partiellement limité par les instruments internationaux qui garantissent à tout individu, d'une part, la reconnaissance de sa personnalitéjuridique, et d'autre part, la jouissance des droits de l'homme. ${ }^{105}$.

Les États, donc, sont en principe libres pour restreindre la capacité de jouissance de certains droits de certaines catégories de personnes, notamment des étrangers. Même les documents internationaux protégeant les droits de l'homme n'imposent pas l'égalité de traitement entre les nationaux et les non-nationaux et reconnaissent les prérogatives des États de créer des distinctions concernant soit le droit privé, soit le droit public.

Le droit brésilien distingue les concepts de personnalité, capacité de droit et capacité de fait. La personnalité est l'aptitude à acquérir des

de travailleurs brésiliens (« La Constitution assure aux travailleurs les droits suivants, ainsi que d'autres qui, dans les termes de la loi, visent à l'amélioration de leur condition sociale: (...) XII - la fixation de pourcentages d'employés brésiliens dans les services publics donnés en concession et dans les établissements de certaines branches commerciales et industrielles »), la Constitution actuelle est silencieuse par rapport à la question.

104 V. Jacob Dolinger, Das Limitações ao Poder de Expulsar Estrangeiros. In : Estudos em Homenagem ao Professor Haroldo Valladão (1983).

105 Marie-Laure Niboyet et Géraud de Geouffre de la Pradelle, Droit International Privé 639 (2007). 
droits et contracter des obligations dont toute personne est dotée ${ }^{106}$. Ce concept a deux sens techniques: le premier se rapporte à la qualité de la personne pour être sujet de droits, alors que le deuxième concerne le bien juridique inhérent à la dignité humaine ${ }^{107}$. La capacité de droit est la faculté abstraite de la personne de jouir de ses droits. Elle peut ou non, toutefois, être accompagnée de la capacité de fait, qui signifie l'aptitude de l'individu à acquérir des droits et aussi à en jouir ou à les exercer sans assistance ou représentation ${ }^{108}$.

La capacité de l'étranger peut être partiellement limitée en quelques circonstances. Il s'agit, parmi autres, des restrictions qui sont imposées par la loi, en ce qui concerne des situations déterminées, des dérogations ponctuelles au principe de l'assimilation des étrangers aux nationaux en droit privé, telles que l'interdiction d'avoir des propriétés dans certaines régions ou d'être actionnaire d'une société. Ces limites, donc, n'affectent pas la personnalité juridique de l'étranger, vu que le droit d'être reconnu en tant que personne devant la loi ne lui est pas nié.

La capacité pour exercer un droit (capacité de fait) est la capacité d'agir en son propre nom dans la sphère juridique. Toute personne domiciliée dans le pays 1 'a ${ }^{109}$, indépendamment de sa nationalité, à l'exception des individus suivants : les mineurs de 18 ans; les handicapés mentaux qui n'ont pas le discernement nécessaire pour pratiquer les actes de la vie civile; ceux qui, bien que par une cause transitoire, ne peuvent pas exprimer leur volonté ; les ivres naturels; les toxicomanes ; ceux qui ont leur discernement réduit par déficience mentale; les exceptionnels, qui n'ont pas eu un développement mental complet; et les prodigues ${ }^{110}$.

Ces règles de capacité n'affectent que ceux qui sont domiciliés au Brésil; si un étranger est domicilié dans un autre pays et a besoin d'exercer un droit au Brésil, sa capacité pour cela sera régie par les lois du pays de son domicile. Ainsi, tout étranger domicilié au Brésil qui a plus de 18 ans et qui n'a aucune des caractéristiques énumérées ci-dessus peut, sans aucune autre formalité, signer un contrat, se marier ou contracter avec quelqu'un au Brésil. Ainsi, on peut entrevoir deux situations différentes: (1) une personne qui a la personnalité juridique et la capacité d'acquérir un droit spécifique peut, selon les lois du pays de son domicile, ne pas avoir la capacité pour exercer ce droit; ou (2)

106 Caio Mário da Silva Pereira, Instituições de Direito Civil, v. 1, 213-14 (2004).

107 Gustavo Tepedino, Temas de Direito Civil 26 (2004).

108 Caio Mário da Silva Pereira, Instituições de Direito Civil, v. 1, 263 (2004).

109 Au Brésil, la capacité d'exercer un droit est réglée pa la loi du domicile de la personne concernée (Loi d' Introduction aux Normes du Droit Brésilien: «Article 7 : La loi du pays où la personne est domiciliée détermine les règles sur le commencement et la fin de la personnalité, le nom, la capacité et les droits de famille »).

110 Code Civil, articles 5 e 6. 
une personne domicilié au Brésil qui a la personnalité et la capacité d'exercice de droits selon la loi brésilienne peut ne pas avoir sa capacité reconnue pour acquérir un droit spécifique, selon la lex fori, du fait qu'il est étranger. Dans ce cas, l'individu ne pourra pas exercer ledit droit, alors que dans le premier exemple il pourra, mais représenté par une autre personne.

Somme toute, même ceux qui ont les deux capacités susmentionnées (de droit et de fait) peuvent être privés de l'exercice de certains droits au Brésil, dû à une interdiction spécifique contenue dans la Constitution ou dans la législation ordinaire. Autrement dit, l'étranger peut subir quelques limitations en certaines matières, telles que celles concernant l'acquisition d'immeubles situés dans des régions rurales ${ }^{111}$, de navires brésiliens ${ }^{112}$ ou de moyens de communication ${ }^{113}$. Cependant, ces restrictions aux droits des étrangers sont des exceptions à la règle et qui sont nécessairement justifiées par d'autres principes invoqués par la politique constitutionnelle.

\section{Accès à la justice}

Conformément à ce qu'on avait mentionné ci-dessus, à tout étranger, résident ou non au Brésil, est garanti le droit d'accéder à nos tribunaux pour obtenir l'assistance juridictionnelle, soit en tant que demandeur, soit en tant que défendeur ${ }^{114}$. Toutefois, le Code de Procédure Civile exige une caution à tout plaideur qui n'est pas domicilié au Brésil et qui n'y a pas de biens, soit-il national ou étranger ${ }^{115}$. Aux étrangers résidents est garanti le droit d'être assisté par des avocats publics, s'ils ne peuvent pas en contracter un ${ }^{116}$.

111 Constitution de 1988, article 140; Loi n 5.709/1971; Decret n 74.965/1974.

112 Constitution de 1998, article 178, § $2^{\circ}$.

113 Constitution de 1998, article 222.

114 V. Cândido Rangel Dinamarco, Sobre a tutela jurisdicional ao estrangeiro 169. In : Revista Forense, v. 357, sept.-oct./2001: "Il n'existe pas, à ce propos, de restriction en référence aux étrangers, voire lorsqu'ils ne sont résidents du territoire national, compte tenu de l'amplitude et de la mens des garanties constitutionnelles des doits fondamentaux; l'origine du sujet est un facteur qui ne peut pas être pris en considération, sous peine d'infraction directe au contenu de l'article 3, alinea IV, de la Constitution Fédérale et au système de garanties entièrement considéré " ("Não existe a esse propósito uma só restrição em referência aos estrangeiros, ainda quando não residentes no território nacional, em vista da amplitude e da mens das garantias constitucionais dos direitos fundamentais; a origem do sujeito é fator que não pode ser levado em consideração, sob pena de infração direta ao disposto no art. $3^{\circ}$, inciso $\mathrm{IV}, \mathrm{da}$ Constituição Federal e ao sistema de garantias como um todo »).

115 Code de Procédure Civile, article 845.

116 Constitution de 1988, article 5, LXXIV ; Loi n 1.060/1950. 


\section{Participation à la vie économique}

La règle quant aux droits privés, c'est l'assimilation entre les nationaux et les étrangers. L'ancien Code Civil Brésilien, de 1916, contenait une norme qui expressément établissait le principe ${ }^{117}$, mais qui n'a pas été reproduite dans le Code Civil, de 2002. Toutefois, la tendance du droit conventionnel est la consécration de ladite règle, tout en assimilant en droit civils, en règle, les nationaux et les étrangers ${ }^{118}$. La règle admet, toutefois, des dérogations ponctuelles auxquelles même les documents internationaux de protection des droits de l'homme ne s'opposent pas.

Ainsi le Pacte des Nations Unies sur les Droits Économiques, Sociaux et Politiques, dans son article 2 (3) établit une exception expresse à la règle de non-discrimination et par conséquent au principe de l'assimilation. Elle détermine que, quant aux droits économiques, les pays en développement peuvent exclure les non-nationaux de la jouissance desdits droits.

La règle, c'est que les activités économiques qui ne sont pas permises aux étrangers ordinaires peuvent l'être par des traités bilatéraux. Par conséquent, les droits qu'ils accordent ne seront pas garantis à tout individu, mais seulement aux nationaux de certains États, c'est-à-dire aux nationaux des pays qui sont parties à ces traités.

La plupart des conventions internationales sur les droits de l'homme garantissent le droit à la propriété privée, mais soumis à des limitations ${ }^{119}$. D'un côté, des instruments internationaux consacrent aussi ce droit dans le contexte de la protection contre l'expropriation. De l'autre côté, le droit pour l'étranger d'acquérir des propriétés n'est pas garanti sans limitation. Ainsi, un étranger peut être empêché d'acquérir une propriété si l'intérêt public l'exige. Une fois acquise avec la permission des lois locales, toutefois, la propriété ne pourra pas être expropriée sans indemnisation ${ }^{120}$. Au niveau international, donc, la

117 Article 3 : « la loi ne fait pas de distinction entre nationaux et étrangers quant à l'acquisition et à la jouissance des droits civils ».

118 Code Bustamante, article 1 ; Convention de la Havane, article 5.

119 Déclaration Américaine de Droits et Devoirs de l'Homme, article 23; Déclaration Universelle, article 17; $1^{\text {er }}$ Protocole de la Convention Euopéenne, article $1^{\text {er }}$; Convention Américaine, article 21; Charte Africaine, article 14.

120 Des questions concernant les distinctions entre étrangers et nationaux en ce qui concerne l'expropriation ont été débattues. Par rapport aux distinctions fondées uniquement sur le fait que l'individu est étranger, est-ce qu'elles heurteraient la régle de droit international qui interdit la discrimination, ou autrement est-ce qu'elles pourraient être considérées parmi les exceptions admises par le droit international - c'est-à-dire la sécurité nationale, les intérêts nationaux ou l'ordre public -? Je crois que, si une règle comporte des distinctions fondées sur le domicile et sur la résidence, elle peut éventuellement être légale et valide, mais celles fondées uniquement su la condition nationale de l'individu ne le peuvent pas. En relation aux standards 
protection n'est garantie qu'à l'usage et au profit de la propriété une fois acquise, mais elle ne l'est pas quant à son acquisition, d'après la plupart des instruments internationaux ${ }^{121}$.

Les étrangers admis au pays en tant que résidents permanents, résidents temporaires ${ }^{122}$ et réfugiés doivent $s^{\prime}$ enregistrer dans les 30 jours après leur entrée. Ceux qui sont enregistrés au Brésil reçoivent une carte d'identité ${ }^{123}$, document qui doit être présenté pour obtenir la permission pour pouvoir travailler ${ }^{124}$.

La Constitution Brésilienne interdit aux étrangers de s'engager dans certaines activités. Ils ne peuvent pas être propriétaires ou contrôler les embarcations brésiliennes ${ }^{125}$; être propriétaire des moyens de communication ${ }^{126}$; obtenir des concessions pour la minoration ${ }^{127}$; obtenir des ressources hydriques et d'autres ressources naturelles ${ }^{128}$.

\section{E. Droits politiques}

Les droits politiques sont ceux qui garantissent à l'individu le pouvoir de participer directement ou indirectement à l'établissement ou à l'administration du gouvernement ${ }^{129}$. Ils ne sont pas uniquement liés à la gestion de sujets de l'État et à l'exercice des fonctions de responsabilité pour la conduite du gouvernement, mais aussi au choix de ceux qui vont gérer l'État et à la détermination ou au contrôle de la politique publique d'un pays.

Sont compris dans cette définition les droits d'exercer un service public en général; de voter et d'être élu ${ }^{130}$; d'exercer des fonctions spécifiques

de compensation, v. Patrick M. Norton, A Law of the Future or a Law of the Past? Modern Tribunals and the International Law of Expropriation, 85 Am.J.Int'l Law 474 (1991).

121 V. Dennis Campbell e David S. Tenzer, Alien Acquisition of Real Property: A Practitioner's Perspective. In: Legal Aspects of Aliens Acquisition of Real Property 6-7 (1980): « (...) the well-established principle of international law that a nation may absolutely prohibit an alien from taking, holding or owning land within its sovereign territory. Because an alien does not necessarily have any rights in this regard, it follows that whatever rights are granted may be conditioned as the sovereign authority chooses. Furthermore, any economic activity conducted within the territorial borders can be similarly regulated or conditioned »).

122 Ceux qui sont mentionnés au Statut de l'Étranger, article 13, I, IV - VII.

123 Statut de l'Étranger, article 30.

124 Statut de l'Étranger, article 30.

125 Decret-loi n 499/1969; Decret-loi n 670/1969, article 3.

126 Constitution de 1988, article 178, § 2 .

127 Constitution de 1988, article 222.

128 Constitution de 1988, article $176, \S 1^{\circ}$.

129 Black's Law Dictionary, Direito Político. V. aussi José Afonso da Silva, Curso de Direito Constitucional Positivo 344 (2005).

130 Ces trois premiers droits sont les droits politiques classiques, les droits politiques fondamentaux et originaux, en opposition aux autres droits, définis comme droits politiques dérivés. 
dans le Pouvoir Exécutif; de participer aux jurys et aux forces armées.

En règle générale, les droits politiques ne sont garantis qu'aux citoyens. La citoyenneté est un concept de droit interne, en ne s'appliquant qu'aux individus nationaux d'un certain État qui sont dans la jouissance pleine des droits politiques. Citoyen n'est pas un synonyme de national: pour être citoyen, il faut avoir des droits politiques, de manière que tout national ne l'est pas ${ }^{131}$.

La citoyenneté tient en considération le lien de droit public entre l'individu et l'État. Il faut rappeler que, toutefois, son concept diffère de celui de la nationalité dans la mesure où la citoyenneté présuppose la capacité de l'individu d'influer sur la formation de la volonté nationale. Pour autant, divers droits sont reconnus (droit de liberté, d'égalité etc.) comme nécessaires à l'exercice de droits politiques actifs et passifs ${ }^{132}$. Ainsi, dans la sphère des droits politiques, l'existence de distinctions entre les citoyens et les étrangers est largement acceptée ${ }^{133}$ et la règle, au contraire de celle en droit privé, c'est que les étrangers sont privés des droits politiques.

Ainsi, seulement les brésiliens peuvent voter aux élections fédérales, des États et des Municipalités ${ }^{134}$ et seulement les nationaux peuvent concourir à des postes publiques ${ }^{135}$. Il s'agit d'une traduction de la notion d'autodétermination des peuples. Toutefois, le fait que les étrangers n'ont pas ces droits est basé sur la théorie selon laquelle seulement les citoyens peuvent participer du gouvernement d'un pays ${ }^{136}$;

131 Charles Gordon e Harry N. Rosenfield, Immigration Law and Procedure, observent que ceratins citoyens ne jouissent pas entièrement des droits politiques, tels que les mineurs (Oregon v. Mitchell, 400 U.S. 112 (1970)), les femmes (Minor v. Happersett, 88 U.S. 162, 22L. Ed. 627 (1874)), les personnes condamnées pour certains crimes et résidentes dans District de Columbia (Plan de Réorganisation $n^{\circ} 3$ de 1967, 5 USC App). Il faut souligner que cela ne veut pas dire que certains citoyens ne jouissent pas de droits politiques, mais que certains nationaux ne sont pas citoyens.

132 T.H. Marshall,. Cidadania, Classe e Status, Trad. Meton Porto Gadelha (1967). José Murilo de Carvalho, Cidadania no Brasil: o longo caminho (2001); Ricardo Lobo Torres, A Cidadania Multidimensional na era dos direitos. In : Ricardo Lobo Torres (Org.), Direitos Fundamentais (2001). 133 Paul Lagarde, in Studi Emigrazione. Études Migrations 10 (1978) souligne l'idée que le refus de certains droits politiques aux étrangers est une tradition de la plupart de démocraties et dit: « le refus de tout droit politique aux étrangers est un tabou sur lequel se sont constitués la plupart des regimes democratiques du monde occidental ». Francis Delpérée, Les Droits Politiques des Étrangers $3 \mathrm{n}^{\circ} 1$ (Que sais-je?, 1995). V. encore Gilmar Ferreira Mendes, Direito de Nacionalidade e Regime Jurídico do Estrangeiro 17. In : Direito Público, $\mathrm{n}^{\circ} 14$, out.-dez./2006.

134 Constitution de 1988, article $14, \S 2^{\circ}$.

135 Constitution de 1988 , article $14, \S 3^{\circ}$, I.

136 V. Déclaration Universelle de Droits de l'Homme, articles 20 (1 et 2); Pacte International sur les Droits Civils et Politiques, article 25 ; Convention Américaine sur les Droits de l'Hommes, article 23 ; Déclaration Américaine de Droits et Devoirs de l'Homme, articles XX, XXXII, 
quelques pays néanmoins garantissent certains droits politiques aux étrangers résidents. Ainsi, les nationaux portugais résidant au Brésil y jouissent de certains droits politiques, en raison du traité signé entre les pays et de l'article 12, paragraphe 2, de la Constitution Fédérale. Il faut qu'ils y résident de manière permanente depuis au moins trois ans et qu'ils en fassent la demande à l'autorité compétente ${ }^{137}$.

\section{LE DROIT INTERNATIONAL ET LA CONSTITUTION DE 1988}

La plupart des règles de droit international sont adressées aux États et en général ne peuvent être effectives que si les États eux-mêmes sont disposés à les mettre en œuvre. Ainsi, l'existence de conventions internationales protégeant les droits de l'homme aurait une importance réduite si les États n'accomplissaient pas les obligations qui leur sont imposées par ces textes.

La dernière section de ce travail examinera la posture adoptée par le Brésil en relation à la communauté internationale en ce qui concerne le respect de la condition des étrangers, afin de montrer l'étendue de l'engagement du pays avec les principes internationaux concernant cette matière. La Constitution Brésilienne de 1988 a adopté et/ou a confirmé les principes de droit international suivants, en ce qui concerne le droit des étrangers:

1. Les nationaux ont le droit d'entrer, de vivre, de se déplacer librement et de ne pas être expulsés du territoire du pays ${ }^{138}$. Aux étrangers, par contre, ne sont pas garantis les droits d'entrer, de résider et de se déplacer dans le territoire national; même s'ils obtiennent une permission pour cela, ce privilège pourra être abrogé, conformément à la loi. L'article 5, XV et LI de la Constitution de 1988 confère aux brésiliens le droit d'entrer dans le pays et empêche sa déportation, expulsion ou extradition. 2. Les nationaux et les étrangers ont le droit de quitter le pays ${ }^{139}$. L'article 5, XV de la Constitution de 1988 n'impose pas de limites au droit de quitter le pays.

XXIV et XXXVIII ; Convention de la Havane sur la Condition des Étrangers, article 7 (1928). 137 Traité de l'Amitié, Coopération et Consulte, entre la République Fédérative du Brésil et la République Portugaise, article 17 (1).

138 Selon la Déclaration Universelle de Droits de l'Homme, article 13 (2) ; Convention Américaine sur les Droits de l'Homme, article 22.5 (1969); Déclaration Américaine des Droits et Devoirs de 1'Homme, article VIII (1948) ; Convention Européenne de Droits de l'Homme, $4{ }^{\mathrm{e}}$ Protocole, article, article 3 (1) (1968); Convention de la Havane sur la Condition des Étrangers, article 6 (1928).

139 Fondé sur la Déclaration Universelle, article 13 (2) ; le Pacte International sur les Droits Civils et Politiques, article 12 (2) (1966) ; la Convention Européenne de Droits de l'Homme, 4' Protocole, article 2; et sur la Convention Américaine sur les Droits de l'Homme, article 22, (3 et 4). 
3. Tous doivent avoir le droit à la vie en famille ${ }^{140}$. L'article 5 de la Constitution de 1988 et le Statut de l'Étranger prennent en considération, lorsqu'ils traitent de l'entrée et de l'expulsion des étrangers, l'existence d'une famille brésilienne de l'étranger. 4. Tous doivent avoir le droit à la liberté d'association ${ }^{141}$. Ce concept inclut la liberté de créer une association et de s'associer à une déjà existent; le droit de demeurer associé et d'être actif dans l'organisation associative; la liberté de s'associer ou de quitter l'organisation; la liberté de grève. Ces droits sont prévus par les articles 8 et 9 de la Charte de 1988.

5. Le droit à l'accès à la justice doit être garanti à tous ${ }^{142} \cdot \mathrm{Ce}$ droit est prévu dans l'article 5, XXXV de la Constitution de 1988.

Enfin, les droits qui sont niés aux étrangers au Brésil par la Constitution de $1988^{143}$ - le droit de participer à la vie politique de l'État et l'accès aux postes publiques - sont des droits dont la négation à l'étranger est permise par le droit international ${ }^{144}$.

Ainsi, bien que le Brésil n'ait pas ratifié beaucoup de conventions internationales à propos des droits des étrangers, la Constitution de 1988 a réaffirmé l'engagement du pays avec les principes fondamentaux établis par le droit international dans le champ de la protection à l'étranger, et les restrictions à ses droits, y compris celui d'entrer et de séjourner au pays, se justifient par d'autres objectifs établis dans la Constitution, de manière qu'il n'y a pas de discrimination arbitraire.

\section{RÉFERÉNCES}

BAPTISTA, L.O. O Estrangeiro: Reflexões para a Constituinte. In: DOLINGER, J. ; FALCÃO, A. P. A nova Constituição e o Direito Internacional. $1^{\text {a }}$ Ed. Rio de Janeiro : Livraria F. Bastos, 1987.

140 Fondé sur l'article 16 (1) de la Déclaration Universelle ; article 8 de la Convention Américaine sur les Droits de 1'Homme de 1950 ; et sur l'Acte Final de la Conférence de Helsinki sur la Sécurité et la Coopération en Europe de 1975.

141 Article 20 (1) de la Déclaration Universelle de Droits de l'Homme ; article 22 du Pacte International des Nations Unies sur les Droits Civils et Politiques ; article 11 de la Convention Européenne de Droits de l'Homme, article 16 de la Convention Américaine sur les Droits de l'Homme ; Conventions de l'Organisation Internationale du Travail numéros 87 et 98.

142 Article 10 de la Déclaration Universelle de Droits de l'Homme ; articles 2 (3) (1) et 13 du Pacte International des Nations Unies sur les Droits Civils et Politiques ; article 6 de la Convention Européenne de Droits de l'Homme .

143 Constitution de 1988 , articles $12, \S 3^{\circ}$ e 14.

144 Articles XX, XXXII, XXXIV et XXXVIII de la Déclaration Américaine de Droits et Devoirs de l'Homme ; article 23 de la Convention Américaine sur les Droits de l'Homme (1969) ; article 25 du Pacte International des Nations Unies sur Droits Civils et Politiques ; article 21 (2 et 3) de la Déclaration Universelle de Droits de l'Homme. 
BASTOS, C. Curso de Direito Constitucional. 16 ${ }^{\text {a }}$ Ed. São Paulo: Saraiva, 1999.

BATALHA, W.C. Direito Internacional Privado. V. 2. $2^{\mathrm{a}}$ Ed. São Paulo: RT,1977.

BORCHARD, Edwin. The Minimus Standard of the Treatment of Aliens. American Society of International Law - Proceedings, Washington, v. 56, 1939.

CAMPBELL,Dennis ;TENZER, D. S. Alien Acquisition of Real Property: A Practitioner's Perspective. In: . Legal Aspects of Aliens Acquisition of Real Property. $1^{\text {a }}$ Ed. Berlim:Springer, 1980.

CARVALHO, A. D. Situação Jurídica do Estrangeiro no Brasil. $1^{\text {a }}$ Ed. São Paulo, Sugestões Literárias, 1976.

CARVALHO, J.M.C. Cidadania no Brasil: o longo caminho. $1^{\text {a }}$ Ed. Rio de Janeiro : Civilização Brasileira, 2001.

CARVALHO,K. G. C. Direito Constitucional. $13^{\text {a }}$ Ed. Belo Horizonte: Del Rey, 2007.

DELPÉRÉE, Francis. Les Droits Politiques des Étrangers 3. Paris: Presses Universitaires de France, 1995.

DINAMARCO, C.R.1. Sobre a tutela jurisdicional ao estrangeiro. Revista Forense, Rio de Janeiro, v. 357, sept/oct. 2001.

DOLINGER, J. Direito Internacional Privado: Parte Geral.11 a Ed. Rio de Janeiro : Editora Forense Jurídica - Grupo Gen, 2014.

DOLINGER, J. Das Limitações ao Poder de Expulsar Estrangeiros. In: VALLADÃO, H. Estudos em Homenagem ao Professor Haroldo Valladão. Rio de Janeiro : Livraria Freitas Bastos, 1983.

DOLINGER, J. Comentários à Constituição de 1988. Rio de Janeiro. Livraria Freitas Bastos, 1990.

FERREIRA FILHO,M. G. Comentários à Constituição Brasileira. $5^{\mathrm{a}}$ Ed. São Paulo: Saraiva, 1984.

FERREIRA, M. G. F. Direito Humanos Fundamentais. $6^{a}$ Ed. São Paulo: Saraiva, 2004.

FRANCA, L. R. 34 a Enciclopédia Saraiva do Direito: Estado Político, Execução das ações. São Paulo: Saraiva, 1977.

FRAGA, M. O novo Estatuto do Estrangeiro Comentado. $1^{\text {a }}$ Ed. Rio de Janeiro: Forense, 1985.

GOODWIN-GILL, G. International Law and the Movement of Persons Between States. 1 ${ }^{a}$ Ed. Ofxord: Oxford University Press, 1978. 
GOODWIN-GILL, G. The Refugee in International Law. $3^{\mathrm{a}} \mathrm{Ed}$. Oxford : Clarendon Press, 1983.

MACDONALD,I. Immigration Law and Practice in the UK 1. $2^{\mathrm{a}} \mathrm{Ed}$. London: Butterworths, 1987.

MARSHALL,T.H. Cidadania, Classe e Status. $1^{\text {a }}$ Ed. Rio de Janeiro: Zahar Editores, 1967.

MELLO, C. A. Direito Internacional Público. $6^{\text {a }}$ Ed. Rio de Janeiro: Livraria Freitas Bastos, 1979.

MENDES,G. Direito de Nacionalidade e Regime Jurídico do Estrangeiro. Direito Público, Brasília, nº 14, out/dez.2006

MIRANDA, P. 4 Comentários à Constituição de 1967 com a Emenda n¹ de 1969. 1974.

MORAES,Alexandre. Direito Constitucional. 20 Ed. São Paulo, Atlas, 2006.

NIBOYET,Marie-Laure; PRADELLE, G. G. P. Droit International Privé. $1^{\mathrm{a}}$ Ed. Paris : LGDJ, 2007.

NORTON, P.M.A. Law of the Future or a Law of the Past? Modern Tribunals and the International Law of Expropriation, 85 Am.J.Int'l Law, 1991.

OPPENHEIM,L. International Law. $7^{\mathrm{a}}$ Ed. Michigan: Longmans, 1955.

PEREIRA, C.M.S. Instituições de Direito Civil. Vol. 1. 20ª Ed. Rio de Janeiro, Forense, 2004.

PIOVESAN, F. O Direito de Asilo e a Proteção Internacional dos Refugiados. In: ARAÚJO,N ; ALMEIDA, G. A. (Coord.). O Direito Internacional dos Refugiados Brasileiros. Rio de Janeiro: Renovar, 2001.

PLENDER,R. International Migration Law. $2^{\mathrm{a}}$ Ed. Dordrecht: Martinus Nijhoff Publishers, 1988.

POGREBINSCHI, T.. O direito de asilo e a Constituinte de 1987-88. In: ARAÚJO,N ; ALMEIDA, G. A. (Coord.). O Direito Internacional dos Refugiados Brasileiros. Rio de Janeiro: Renovar, 2001.

RAMOS, M. O Novo Direito Português da Nacionalidade. In : Correia, A. F. Homenagem ao Prof. Antonio Arruda Ferrer Correia. Coimbra: Universidade de Coimbra, 1986.

RAMOS, M. La Double Nationalité D'après le Droit Portugais. Boletim da Faculdade de Direito de Coimbra, Coimbra, v. 59, 1983. 
RESEK, J. F. Direito Internacional Público: Curso elementar. $9^{\mathrm{a}} \mathrm{Ed}$. São Paulo : Saraiva, 2002.

SILVA, J. A. Curso de Direito Constitucional Positivo. 24a Ed. São Paulo: Malheiros, 2005.

SOARES, G. F. S. Os Direito Humanos e a Proteção dos Estrangeiros. Revista de Informação Legislativa, Brasília n 164, abr/jun. 2004.

SOARES, G.F.S. Os estrangeiros e as Atividades a eles Vedadas ou Restringidas Proibição Constitucional a Disc riminação pela Lei Ordinária de Brasileiros Naturalizados. In: DOLINGER, J. ; FALCÃO, A. P. A nova Constituição e o Direito Internacional. $1^{\text {a }}$ Ed. Rio de Janeiro : Livraria F. Bastos, 1987.

TENORIO, O. Direito Internacional Privado. Vol.1. 11 a Ed. Rio de Janeiro, Freitas Bastos, 1976.

TEPEDINO, G. Temas de Direito Civil. $3^{\text {a }}$ Ed. Rio de Janeiro: Renovar, 2004.

TIBURCIO, C. Nationality and the Status of Aliens in the 1988 Brazilian Constitution. In: DOLINGER, J.; ROSENN, K. S. A Panorama of Brazilian Law. $1^{a}$ Ed. Miami : Nort - South Center, 1992.

TIBURCIO, C. The Human Rights of Aliens under International and Comparative Law. $1^{\mathrm{a}}$ Ed. Haia: Martinus Nijhoff Publishers, 2001.

TIBURCIO, C. A Nacionalidade à Luz do Direito Internacional Brasileiro. In. TIBURCIO, C.; BARROSO, L.R. Direito Constitucional Internacional. $1^{\text {a }}$ Ed. Rio de Janeiro: Renovar, 2013.

TIBURCIO, C. A Extradição no Brasil: análise à luz da jurisprudência e do direito comparado. In. TIBURCIO, C.; BARROSO, L.R. Direito Constitucional Internacional. $1^{\text {a }}$ Ed. Rio de Janeiro: Renovar, 2013.

TORRES,R. L. A Cidadania Multidimensional na era dos direitos. In: TORRES,R. L.(Org.). Direitos Fundamentais. $2^{\text {a }}$ Ed. Rio de Janeiro: Renovar, 2001.

VALLADÃO, H. Direito Internacional Privado. V. 1. Rio de Janeiro: Freitas Bastos, 1980.

VELLOSO, C. M. S. V.O Direito Internacional e o Supremo Tribunal Federal 6-7. Revista de Direito Administrativo, Rio de Janeiro, v. 229, jul/set.2002. 